\title{
Salvianolic acid B protects against myocardial ischaemia-reperfusion injury in rats via inhibiting high mobility group box 1 protein expression through the PI3K/Akt signalling pathway
}

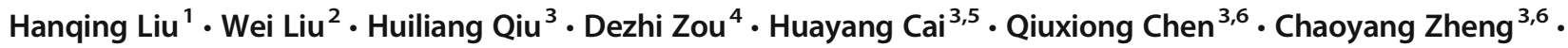 \\ Danping $\mathrm{Xu}^{3,6,7}$
}

Received: 21 February 2019 / Accepted: 23 October 2019 / Published online: 18 December 2019

(C) The Author(s) 2019

\begin{abstract}
Salvianolic acid B (Sal B) has a significant protective effect on myocardial ischaemia-reperfusion (I/R) injury. Therefore, the aims of this study were to determine the effects of Sal B on myocardial ischaemic-reperfusion (I/R) injury in rats and to explore whether its underlying mechanism of cardioprotection occurs through activating the expression of the phosphoinositide 3-kinase/ protein, kinase B (PI3K/Akt) and inhibiting the expression of high mobility group protein 1 (HMGB1). Ninety Sprague-Dawley rats were randomized into five groups: group 1 (sham-operated), group 2 (myocardial I/R), group 3 (low dose of Sal B+I/R), group 4 (high dose of Sal B+I/R), and group 5 (high dose of Sal B+I/R+LY294002, which is a specific PI3k inhibitor). All I/R rats received $30 \mathrm{~min}$ myocardial ischaemia followed by 24-h reperfusion. Cardiac function, infarct size, myocardial injury marker levels, inflammatory response and cardiomyocyte apoptosis as well as Bcl-2, Bax, P-Akt, HMGB1 and TLR4 expression were measured. In the current study, Sal B significantly ameliorated myocardial I/R injury in a dose-dependent manner, ameliorated cardiac function, reduced myocardial infarction size, decreased myocardial injury marker expression, decreased inflammatory responses, reduced apoptosis, activated PI3K/Akt expression and inhibited HMGB1 expression. However, all effects of Sal B were significantly reversed by LY294002. Overall, the present study indicated that Sal B attenuated myocardial I/R injury by activating $\mathrm{PI} \mathrm{K} / \mathrm{Akt}$ and inhibiting the release of $\mathrm{HMGB} 1$ in rats.
\end{abstract}

Keywords Sal B · Myocardial ischaemia/reperfusion injury $\cdot$ PI3K/Akt/HMGB1

\section{Introduction}

Coronary heart disease is a leading cause of death associated with cardiovascular disease. Acute myocardial infarction

Hanqing Liu and Wei Liu contributed equally to this work.

Hanqing Liu and Wei Liu should be considered to share first authorship.

Chaoyang Zheng

zhengchaoyang@medmail.com

$\triangle$ Danping Xu

173227223@qq.com

1 Cardiovascular Department, Guangzhou Hospital of integrated Traditional and West Medicine, Guangzhou 510800, China

2 Geriatrics Department, Tongde Hospital of Zhejiang Province, Hangzhou 310012, Zhejiang, China

3 Second Clinical Medical College, Guangzhou University of Chinese Medicine, Guangzhou 510006, China
(AMI)-associated mortality and morbidity are common worldwide (Frank et al. 2012). Early myocardial reperfusion is the preferred treatment for AMI in most clinical settings (Binder et al. 2015). The rapid restoration of blood flow is a critical

4 Emergency Department, The First Affiliated Hospital, Sun Yat-sen University, Guangzhou 510080, China

5 Internal Medicine Department, Guangdong Provincial Hospital of Chinese Medicine, 2nd Affiliated Hospital of Guangzhou University of Chinese Medicine, Guangzhou 510120, China

6 Cardiovascular Department, Guangdong Provincial Hospital of Chinese Medicine, 2nd Affiliated Hospital of Guangzhou University of Chinese Medicine, Guangzhou 510120, China

7 Guangdong Provincial Academy of Chinese Medical Sciences, Guangzhou 510006, China 
therapeutic strategy. However, abrupt myocardial reperfusion can sometimes exacerbate tissue injury and is called myocardial ischaemia and reperfusion (I/R) injury (Jennings 2013). The mechanism of myocardial $\mathrm{I} / \mathrm{R}$ injury is very complex. There are numerous causes of myocardial I/R injury, such as inflammation, apoptosis, mitochondrial dysfunction and oxidative stress (Braunersreuther et al. 2013; Zhu et al. 2015; Li et al. 2016a, b, Wang, Han and Jia 2018).

Danshen, the dried root of Salvia miltiorrhiza Bunge, is a Chinese medicinal herb. Salvianolic acid B (Sal B) is an active water-soluble component that can be isolated from S. miltiorrhiza Bunge (Chan et al. 2004; Hu et al. 2005; Lam et al. 2007). Sal B exhibited multiple bioactivities, including the reduction of the expression of related inflammatory factors, inhibition of apoptosis and alleviation of oxidative stress (Lv et al. 2015; Zhao et al. 2017). As an abundant bioactive component of $S$. miltiorrhiza, it has been reported that Sal B protects against I/R-induced cerebral injury by reducing cerebral infarct size and improving neurobehavioural functions (Fan et al. 2018). In addition, it has been shown that Sal B has cardiovascular protective effects, due to its potent anti-oxidative capabilities and inhibition of inflammation during cardiovascular injury (Ho and Hong 2011). Additionally, previous studies have demonstrated that Sal B has significant protective effect on myocardial I/R injury by alleviating oxidative stress, reducing calcium overload, improving endothelial function, stabilizing mitochondrial membrane potential and upregulating microRNA-30a (Xue et al. 2014; Deng et al. 2015; Li et al. 2016a, b).

The PI3K/Akt signalling pathway plays an important role in cardioprotection and is involved in regulating cardiomyocyte survival, apoptosis and inflammatory responses (Cao et al. 2013; Yao, Han and Han 2014; Tang et al. 2017). Recently, some studies have reported that endoplasmic reticulum stress-regulated apoptosis and excessive autophagy can be inhibited and the myocardium can be protected from $\mathrm{I} / \mathrm{R}$ injury through the activating PI3K/Akt signalling pathway ( $\mathrm{Li}$ et al. 2018; Yu et al. 2019). HMGB1 is a nuclear protein that is passively released from injured cells (Zhou, $\mathrm{Li}$ and $\mathrm{Mu} 2015$; Ouyang et al. 2016). It has been confirmed that dexmedetomidine protects cardiomyocytes against hypoxia/ reoxygenation-induced necrosis by inhibiting HMGB1mediated inflammation (Chen et al. 2019).

Previous study has shown that anti-inflammatory effect of B-type natriuretic peptide(BNP) postconditioning during myocardial I/R injury is associated with inhibiting HMGB1 expression through activating of PI3K/Akt signalling pathway (Hu et al. 2014). Furthermore, Celastrol and simvastatin have been reported to protect the myocardium against I/R injury by inhibiting HMGB1 expression via activating PI3K/Akt pathway (Han et al. 2015; Tong et al. 2018).

Therefore, we determined whether Sal B could modulate the release of HMGB1 during myocardial I/R injury and speculated that the cardioprotective mechanism of Sal B against myocardial $\mathrm{I} / \mathrm{R}$ injury was through the inhibition HMGB1 expression through activating the PI3K/Akt signalling pathway.

\section{Materials and methods}

\section{Animals}

Ninety male Sprague-Dawley rats $(250-300 \mathrm{~g})$ were obtained from Guangdong Provincial Medicinal Laboratory Animal Center (SCXK (Yue) 2013-0002, Guangzhou, China). All animals in this study were treated in accordance with the Guide for the Care and Use of Laboratory Animals published by the National Institutes of Health (Bethesda, MD, USA) and the Regulations of Experimental Animal Administrations published by the State Committee of Science and Technology of the People's Republic of China. The study protocol was approved by the Animal Care Committee of Guangdong Provincial Hospital of Chinese Medicine. All rats used in this study were allowed free access to food and water and were housed in the Animal Center of Guangdong Provincial Hospital of Chinese Medicine.

\section{Drugs and chemicals}

Sal B (HPLC > 98\%) was purchased from Chengdu Manst Biotechnology Co. Ltd. 2,3,5-Triphenyltetrazolium chloride (TTC) was purchased from MB-CHEM (USA). Haematoxylin (HE) was purchased from Keygen Biological Technology Co. Ltd., Jiangsu, China. T-Akt (11E7), P-Akt (Ser473) and glyceraldehyde-3-phosphate dehydrogenase (GAPDH) (5174) were purchased from Cell Signaling Technology (USA). The HMGB1 and goat anti-rabbit secondary antibodies were purchased from Abcam (USA). Bcl-2 associated $\times$ protein $(\mathrm{Bax})$, Beclin-2 (Bcl-2) and Toll-like receptor 4 (TLR4) were purchased from Proteintech Group, Inc. (USA). LY294002 was purchased from MedChemExpress (China). L-Lactate dehydrogenase (L-LDH), creatine kinase (CK-MB), tumour necrosis factor- $\alpha$ (TNF- $\alpha)$, interleukin-18 (IL-18), interleukin-1 $\beta$ (IL-1 $\beta$ ) and HMGB1 enzyme-linked immunosorbent assay (ELISA) kits were purchased from Wuhan Huamei Bioengineering Co., Ltd. 3,3'Diaminobenzidine(DAB) was purchased from China Hubei Boster Biotechnology Co., Ltd. Terminal deoxynucleotidyl nick-end labelling (TUNEL) kit and phosphatase inhibitor cocktail were purchased from Roche Group, Inc. (Swiss). RIPA lysis buffer was purchased from Beyotime Institute of Biotechnology (China). BCA protein assay kit was purchased from Thermo (USA). Enhanced chemiluminescence reagents (ECL) was purchased from EMD Millipore (USA). 
Sal B was dissolved in $0.9 \%$ sodium chloride $(\mathrm{NaCl})$. Different doses of Sal B were administered through intraperitoneal (i.p.) injection immediately after being dissolved in $0.9 \% \mathrm{NaCl}$ and $30 \mathrm{~min}$ before the myocardial ischaemia models were completed.

\section{Experimental protocols}

The rats were randomly assigned to five groups and prepared for different treatments, based on previous studies with modifications (Xue et al. 2014; Qiao and Xu 2016). Group 1 (sham-operated (Sham, $n=18$ )) was not treated, and rats in this group underwent surgical manipulation without ligating left anterior descending coronary artery (LAD). Group 2 (myocardial $\mathrm{I} / \mathrm{R}(\mathrm{I} / \mathrm{R}, n=18)$ ) rats were ischaemic for $30 \mathrm{~min}$ and reperfused for $24 \mathrm{~h}$. Group 3 (low dose of Sal $\mathrm{B}+\mathrm{I} / \mathrm{R}(\mathrm{Sal}-\mathrm{L}, n=18)$ ) rats received $15 \mathrm{mg} / \mathrm{kg} /$ day Sal B via intraperitoneal injection for 4 days before operation. Group 4 (high dose of Sal B+I/R (Sal-H, $n=18)$ ) rats received $60 \mathrm{mg} / \mathrm{kg} /$ day Sal B via intraperitoneal injection for 4 days before operation. Group 5 (high dose of Sal B+I/R+ LY294002 (Sal-H+LY, $n=18)$ ) rats received $60 \mathrm{mg} / \mathrm{kg} /$ day Sal B via intraperitoneal injection for 4 days before operation and LY294002 (an inhibitor of PI3K, $0.3 \mathrm{mg} / \mathrm{kg}$ ) dissolved in $0.02 \%$ dimethyl sulfoxide via caudal vein injection $30 \mathrm{~min}$ before LAD ligation. All I/R rats underwent myocardial ischaemia for $30 \mathrm{~min}$ followed by reperfusion for $24 \mathrm{~h}$.

\section{Myocardial I/R model in rats}

Rats were anaesthetized with intraperitoneal injection of $10 \%$ chloral hydrate $(350 \mathrm{mg} / \mathrm{kg})$. After the rats were anaesthetized, they were treated with artificial ventilation with a volumecontrolled rodent ventilator (Harvard Apparatus, Model Inspira) at a rate of 60 strokes per minute and monitored with an electrocardiograph. Briefly, left thoracotomy and pericardiotomy were performed, and 7-0 nylon slip-knot sutures were inserted around the LAD. Then, myocardial ischaemia was induced. The myocardial ischaemia rats were monitored, and their condition was confirmed by ST segment elevation in an electrocardiogram (ECG). Then, an I/R injury animal model was induced by ligating the LAD for $30 \mathrm{~min}$ followed by 24-h reperfusion (Samsamshariat, Samsamshariat and Movahed 2005).

\section{Cardiac function assessment}

After the rats were anaesthetized, cardiac function was assessed by cardiac output (CO), left ventricular ejection fraction (LVEF), fractional shortening (FS), stroke volume (SV) and heart rate (HR). These parameters were monitored and digitally processed throughout an echocardiograph using a Visual Sonics Vevo 2100 system (Canada).

\section{Infarct size assessment}

Myocardial infarct size was measured with a $1 \%$ solution of TTC stain. In brief, after $24 \mathrm{~h}$ of reperfusion, the heart was removed and frozen at $-20^{\circ} \mathrm{C}$. Then, each heart was horizontally sliced into 5 -mm slices. These slices were incubated in $1 \%$ TTC in phosphate buffer for $15-20 \mathrm{~min}$ at $37^{\circ} \mathrm{C}$ photographed used a digital camera (Canon, Japan). After TTC staining, the white part represented myocardial infarction area, and the red part represented no infarction. Myocardial infarct size was analysed using ImageJ 1.36 software from the National Institutes of Health. Infarct size was expressed as the following percentage: infarction area/total left ventricular area (INF/LV). The total left ventricular area corresponds to the risk area because global ischaemia was induced (Wu et al. 2014).

\section{ELISA analysis}

After $24 \mathrm{~h}$ of reperfusion, blood samples were collected from the abdominal aorta and centrifuged at $4{ }^{\circ} \mathrm{C}$ for $15 \mathrm{~min}$ at a speed of $1000 \mathrm{~g}$. The supernatant was stored at $-80^{\circ} \mathrm{C}$ until ELISA analysis was performed. The levels of L-LDH, CKMB, TNF- $\alpha$, IL-18, IL-1 $\beta$ and HMGB1 were measured by using commercial ELISA kits following the manufacturer's instructions.

\section{Immunohistochemical staining}

The myocardial tissue specimens of rats were fixed in a $4 \%$ paraformaldehyde solution, dehydrated and embedded in paraffin. The tissue samples were cut into 3 - $\mu$ m-thick sections. These sections were dewaxed with gradient xylenol solutions and repaired with a sodium citrate antigen solution. Endogenous catalase activity was inhibited with $3 \%$ hydrogen peroxide, and the sections were sealed with 5\% Bovine Serum Albumin (BSA) at $37{ }^{\circ} \mathrm{C}$ for $1 \mathrm{~h}$. Then, the samples were incubated overnight with HMGB1 and TLR4 primary antibodies at $4{ }^{\circ} \mathrm{C}$. After rinsing with PBS three times ( 3 min each time), the samples were treated with a biotin goat anti-rabbit secondary antibody solution, incubated at $37{ }^{\circ} \mathrm{C}$ for $30 \mathrm{~min}$ and washed with PBS 3 times ( 3 min each time). In addition, the samples were treated with a DAB dye solution for 1 to 2 min and washed with PBS once. The samples were stained again with $\mathrm{HE}$ for 1 2 min, washed with water, dehydrated with gradient ethanol xylene solutions and sealed with neutral balata. Finally, the distribution of immunopositive cells was evaluated under a light microscope. All the analyses were performed with Image-Pro Plus 6.0 software.

\section{Apoptosis analysis}

Myocardial apoptosis was analysed by using a TUNEL kit following the manufacturer's protocol. In brief, after $24 \mathrm{~h}$ of 
reperfusion, the heart was excised. The anterior wall of the left ventricle of the heart was removed, fixed with $4 \%$ formaldehyde, embedded in paraffin, cut into 5- $\mu \mathrm{m}$ sections and stained with TUNEL. The apoptotic cardiomyocytes of rats were visualized under an optical microscope, and the apoptotic cells were counted using the Image-Pro Plus 6.0 software from the National Institutes of Health. The percentages of TUNEL-positive cells were calculated by dividing the total number of apoptotic cells by the total number of cells in each field.

\section{Western blotting}

The left ventricle tissues of rats were homogenized in $1 \mathrm{ml}$ RIPA lysis buffer containing $1 \mathrm{mM}$ phenylmethanesulfonyl fluoride and $1 \%$ phosphatase inhibitor cocktail and then centrifuged at $14000 \mathrm{rpm}$ at $4{ }^{\circ} \mathrm{C}$ for $15 \mathrm{~min}$. The supernatants were collected and used for western blot assays. Protein concentrations were quantified by a BCA protein assay kit. Proteins $(15-20 \mu \mathrm{g})$ were separated by $10-12 \%$ sodium dodecyl sulfate polyacrylamide gel electrophoresis (SDS-PAGE) and then transferred onto polyvinylidene fluoride membranes (EMD Millipore, USA). Following blocking for $2 \mathrm{~h}$ with $5 \%$ skim milk at room temperature, the membranes were incubated with primary antibodies overnight at $4{ }^{\circ} \mathrm{C}$. After washing three times with TBST, the membranes were incubated with secondary antibodies at room temperature for $1.5 \mathrm{~h}$. The protein bands were detected by ECL and the results were quantified by Image Lab 5.2.1 software (Bio-Rad, Hercules, USA). The primary antibodies used included T-Akt (11E7), P-Akt (Ser473), GAPDH (5174), HMGB1, Bcl-2 and Bax.

\section{Statistical analysis}

All the data are expressed as mean \pm SD. SPSS 17.0 software was used for statistical analysis. Among the statistical analyses, the normal distribution and homogeneity of variance measurements were measured by one-way ANOVA, and the test level was alpha $=0.05$. At $P<0.05$, the difference was considered statistically significant. The Kruskal-Wallis test was used for data with non-homogeneous variance. $T$ test or rank sum test was used for comparisons between two groups.

\section{Result}

\section{Sal B ameliorated cardiac function}

The values of HR, SV, LVEF, FS and CO in each group are shown in Fig. 1. There were no significant differences among the HR values of the groups. The SV, LVEF, FS and CO values of the $\mathrm{I} / \mathrm{R}$ group were significantly decreased compared with those of the sham group $(P<0.05)$. After treatment with
Sal $\mathrm{B}$ in the I/R group, the SV, LVEF, FS and CO values significantly increased $(P<0.05)$. Compared with Sal B-L group, the SV, LVEF, FS and CO values of Sal B-H group significantly increased $(P<0.05)$. However, after adding LY294002 to the Sal-H group, the SV, LVEF, FS and CO values were significantly decreased $(P<0.05)$.

\section{Sal B reduced myocardial infarction size}

As shown in Fig. 2, no myocardial infarction was found in the sham group. Compared with the sham group, rats in the I/R group had clear infarction. Compared with the I/R group, the percentage of myocardial infarction area in the Sal-L and Sal$\mathrm{H}$ groups was significantly decreased $(P<0.05)$. Meanwhile, the percentage of infarction area was significantly decreased in Sal-H group compared with the Sal-L group $(P<0.05)$. These results suggested that Sal B reduced infarction area in a dose-dependent manner. However, the reduction of the percentage of infarction area was abolished by LY294002 treatment in the Sal-H group $(P<0.05)$, and the infarction area following LY294002 treatment was similar to that of I/R group.

\section{Sal B reduced myocardial injury and inflammatory response}

L-LDH, CK-MB, TNF- $\alpha$, IL-18, IL-1 $\beta$ and HMGB1 levels were measured to determine whether Sal B attenuated myocardial I/R injury after $24 \mathrm{~h}$ of reperfusion (Fig. 3). Compared with the sham group, the levels of L-LDH, CK-MB, TNF- $\alpha$, IL-18, IL-1 $\beta$ and HMGB1 in the I/R group were markedly elevated $(P<0.05)$. Compared with the $\mathrm{I} / \mathrm{R}$ group, treatment of the I/R group with Sal B (15 and $60 \mathrm{mg} / \mathrm{kg}$ ) inhibited the increased L-LDH, CK-MB, TNF- $\alpha$, IL-18, IL- $1 \beta$ and HMGB1 levels in a dose-dependent manner $(P<0.05)$. However, the levels of L-LDH, CK-MB, TNF- $\alpha$, IL-18, IL$1 \beta$ and HMGB1 in the I/R group treated with Sal B $(60 \mathrm{mg} / \mathrm{kg})$ were significantly abolished by LY294002 treatment $(P<0.05)$. These results demonstrated that Sal B had a protective effect against myocardial I/R injury and inflammatory response, while LY294002 had a partial reversal effect on myocardial I/R injury $(P<0.05)$.

An immunohistochemical method was used to detect the expression of HMGB1 and TLR4 in each group (Fig. 4). The positive staining for HMGB1 and TLR4 was lower in rats with myocardial $I / R$ injury than in rats without $I / R$ injury. Under light microscopy, myocardial cells with varying amounts of brown granules were HMGB1- and TLR4positive cells while cells without this staining pattern were negative for HMGB1 and TLR4. Compared with the sham group, the number of HMGB1- and TLR4-positive cells were significantly increased in the $\mathrm{I} / \mathrm{R}$ group $(P<0.05)$, while those in the Sal B group were significantly decreased in a dose- 

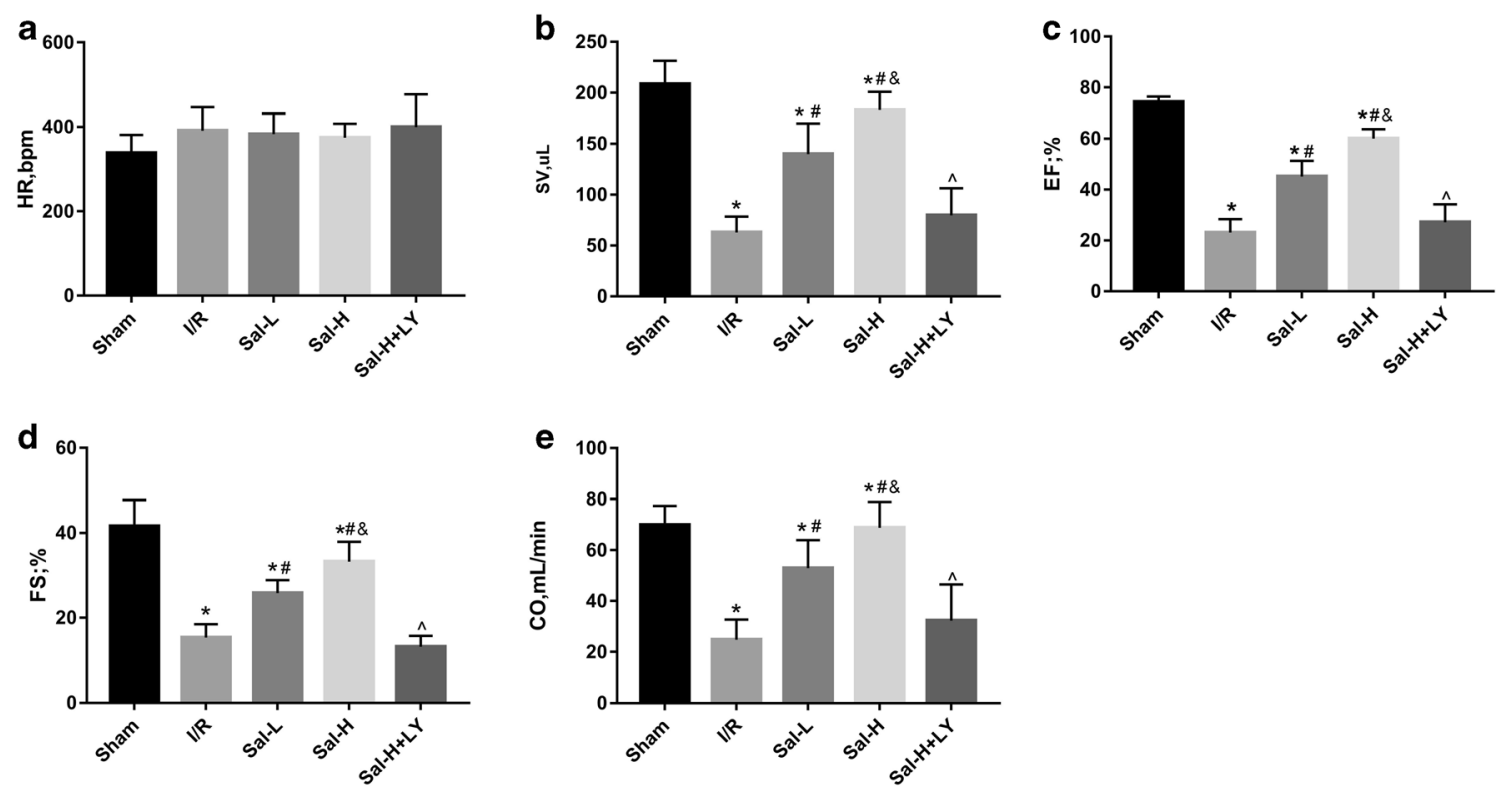

Fig. 1 Cardiac function. Sal B ameliorated cardiac function, but this effect was abolished by LY294002 treatment. The values of HR (a), SV (b), LVEF (c), FS (d) and CO (e) were monitored and digitally processed throughout echocardiography digitally processed throughout an echocardiography. Heart rhythm (HR), cardiac output (CO), ejection fraction (EF), fractional shortening (FS), stroke volume (SV). Sham- operated rats (Sham, $n=18$ ), myocardial ischaemia reperfusion injury rats (I/R, $n=18), \mathrm{I} / \mathrm{R}$ rats treated with low dose of Sal B (Sal-L, $n=18)$, $\mathrm{I} / \mathrm{R}$ rats treated with high dose of Sal B (Sal-H, $n=18)$, Sal-H rats treated with the PI3K inhibitor LY294002 before LAD ligation (Sal-H+LY, $n=$ 18). All data are expressed as mean $\pm \mathrm{SD},{ }^{*} P<0.05$ versus Sham, ${ }^{\#} P<$ 0.05 versus $\mathrm{I} / \mathrm{R},{ }^{\circledR} P<0.05$ versus Sal-L, ${ }^{\wedge} P<0.05$ versus $\mathrm{Sal}-\mathrm{H}$
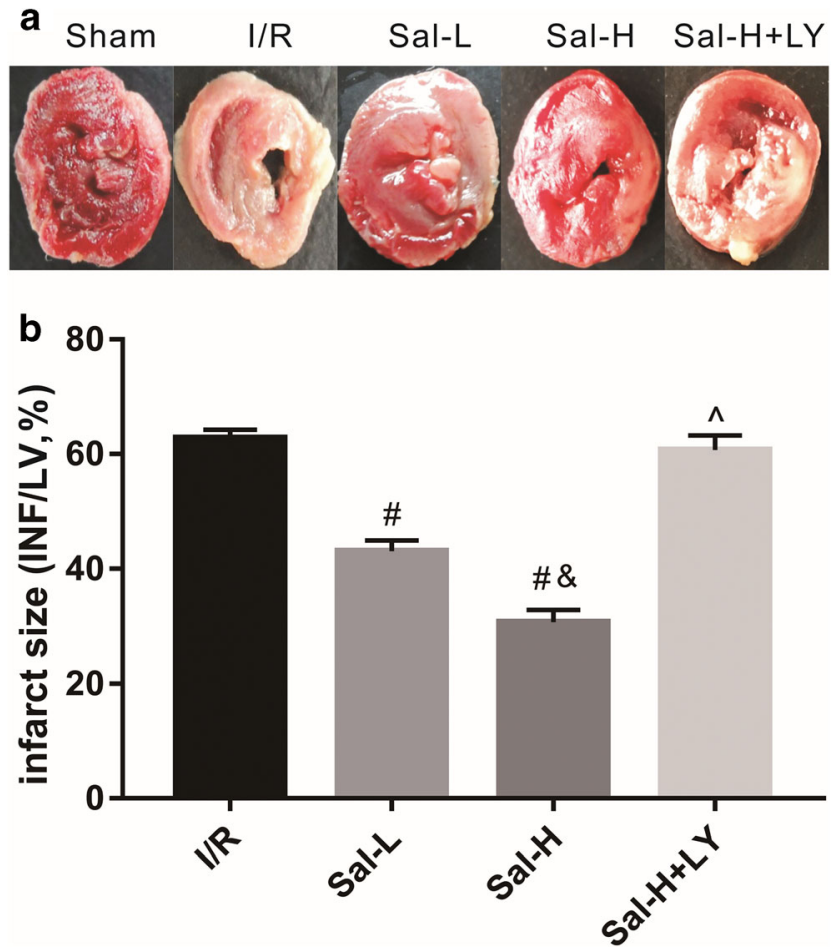

Fig. 2 Myocardial infarct size. Sal B reduced the size of myocardial infarction, but this effect was reversed by LY294002 treatment. Myocardial infarct size was measured with TTC staining, the white part represented myocardial infarction (a) and the myocardial infarct size was analysed (b). All data are expressed as mean $\pm \mathrm{SD},{ }^{\#} P<0.05$ versus I/R, ${ }^{\&} P<0.05$ versus Sal-L, ${ }^{\wedge} P<0.05$ versus Sal-H. INF, infarction area. LV, total left ventricular area dependent manner compared to the $\mathrm{I} / \mathrm{R}$ group $(P<0.05)$. However, the effect of Sal B was reversed by treatment with LY294002 $(P<0.05)$.

\section{Sal B suppressed myocardial apoptosis}

The percentage of cardiomyocyte apoptosis in all experimental groups was assessed by TUNEL staining (Fig. 5a, b). Compared with the sham group, the number of TUNELpositive cells was significantly increased in the I/R group and remarkably reduced in the Sal B treatment group $(P<0.05)$. Moreover, the anti-apoptotic effect of Sal B also occurred in a dose-dependent manner $(P<0.05)$. However, this anti-apoptotic effect was significantly reversed by the administration of LY294002 $(P<0.05)$. Next, we examined the expression of the apoptosis-related proteins Bcl-2 and Bax to further elucidate the underlying mechanism of the antiapoptotic effect of Sal B (Fig. 5c, d). Compared with the sham group, the Bcl-2 expression level and the Bcl-2/bax ratio of the $\mathrm{I} / \mathrm{R}$ group were significantly decreased $(P<0.05)$, while the Bax expression level was remarkably increased $(P<0.05)$. However, after treatment with Sal B, the expression of Bcl-2 and the ratio of $\mathrm{Bcl}-2 / \mathrm{Bax}$ significantly increased and the expression of Bax decreased $(P<0.05)$. These results showed that Sal B could effectively reduce cardiomyocyte apoptosis. In contrast, the anti-apoptotic effect of Sal B was partly reversed by LY294002 treatment $(P<0.05)$. 
Fig. 3 Myocardial injury markers and inflammatory response. Sal B alleviated the level of myocardial injury markers and inflammatory response, while LY294002 has a partial reversal effect. The levels of L-LDH (a) and CK-MB (b) and the expression of TNF- $\alpha(\mathbf{c})$, IL-18 (d), IL-1 $\beta$ (e) and HMGB1 (f) were measured by using enzyme-linked immunosorbent assay (ELISA). All data are expressed as mean $\pm \mathrm{SD}, * P<$ 0.05 versus Sham, ${ }^{\#} P<0.05$ versus $\mathrm{I} / \mathrm{R},{ }^{\&} P<0.05$ versus Sal-L, ${ }^{\wedge} P<0.05$ versus Sal-H
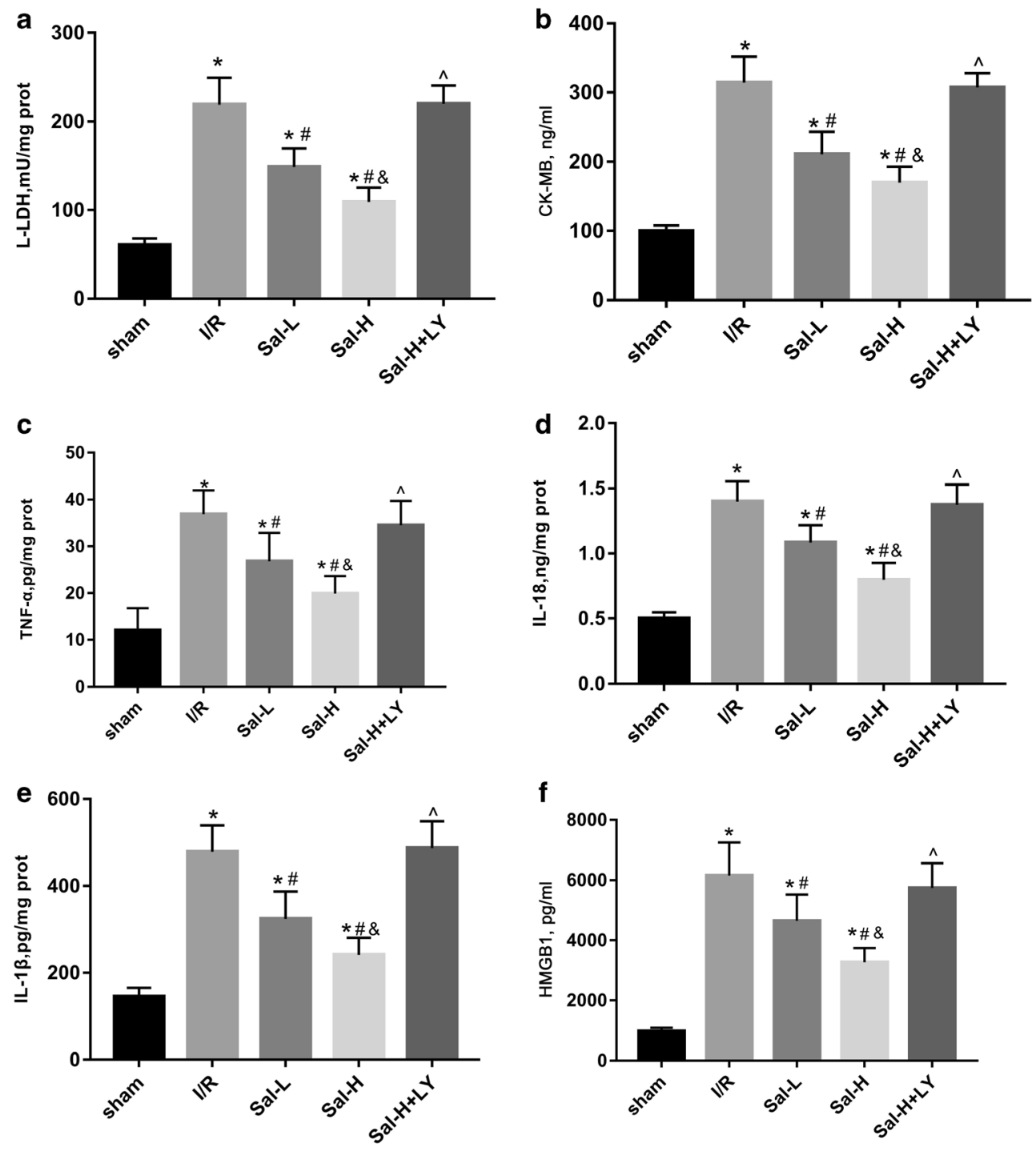

\section{Sal B activated the PI3K/Akt pathway and suppressed HMGB1 expression}

To further investigate the molecular mechanism underlying Sal B-mediated cardioprotection, we determined the expression of P-Akt and HMGB1 protein by western blotting (Fig. 6). Compared with the sham group, the P-Akt protein level was markedly decreased (Fig. 6a, b) and the HMGB1 protein level was significantly increased in the I/R group (Fig. $6 \mathrm{c}, \mathrm{d})(P<0.05)$. However, with Sal B treatment, the decrease in the P-Akt protein level and the increase in the HMGB1 protein level were both inhibited $(P<0.05)$. These results suggest that Sal B plays a cardioprotective role in myocardial $\mathrm{I} / \mathrm{R}$ injury. Moreover, Sal B affected the expression of these proteins in a dose-dependent manner $(P<0.05)$. However, all the effects of Sal B were significantly blocked by treatment with LY294002 $(P<0.05)$. In addition, there was no difference in the T-Akt expression of each group.

\section{Discussion}

S. miltiorrhiza, as a traditional Chinese herb, has been widely used for the treatment of ischaemic heart disease in China (Ho and Hong, 2011), and its therapeutic effect mainly relies on its chemical components. Sal B, one of the active components extracted from S. miltiorrhiza, has been demonstrated to significantly alleviate myocardial I/R injury in a dose-dependent manner (Ji, Tan and Zhu 2000; Xue et al. 2014; Deng et al. 2015).

In this study, Sal B can significantly ameliorate cardiac dysfunction, reduce the release of myocardium enzyme and decrease infarction size, suggesting that Sal B could produce a protective effect in $\mathrm{I} / \mathrm{R}$ rats. This may be related to the effects of Sal B, which can attenuate lung and brain injury in rats through inhibiting apoptosis and inflammation ( $\mathrm{Lv}$ et al. 2015; Zhao et al. 2017). In our findings, it was demonstrated that Sal B treatment can remarkably reduce the infarction size 
Fig. 4 Immunohistochemical detection of HMGB1 and TLR4 expression. Sal B attenuated the expression levels of HMGB1 (a) and TLR4 (b) in rats with myocardial I/R injury, but LY294002 abolished this effect. Immunopositive cells distribution of HMGB1 (c) and TLR4 (d) were evaluated under light microscope. All data are expressed as mean $\pm \mathrm{SD}, * P<0.05$ versus Sham, ${ }^{\#} P<0.05$ versus $\mathrm{I} / \mathrm{R},{ }^{\mathrm{\&}} P<$ 0.05 versus Sal-L, ${ }^{\wedge} P<0.05$ versus Sal-H
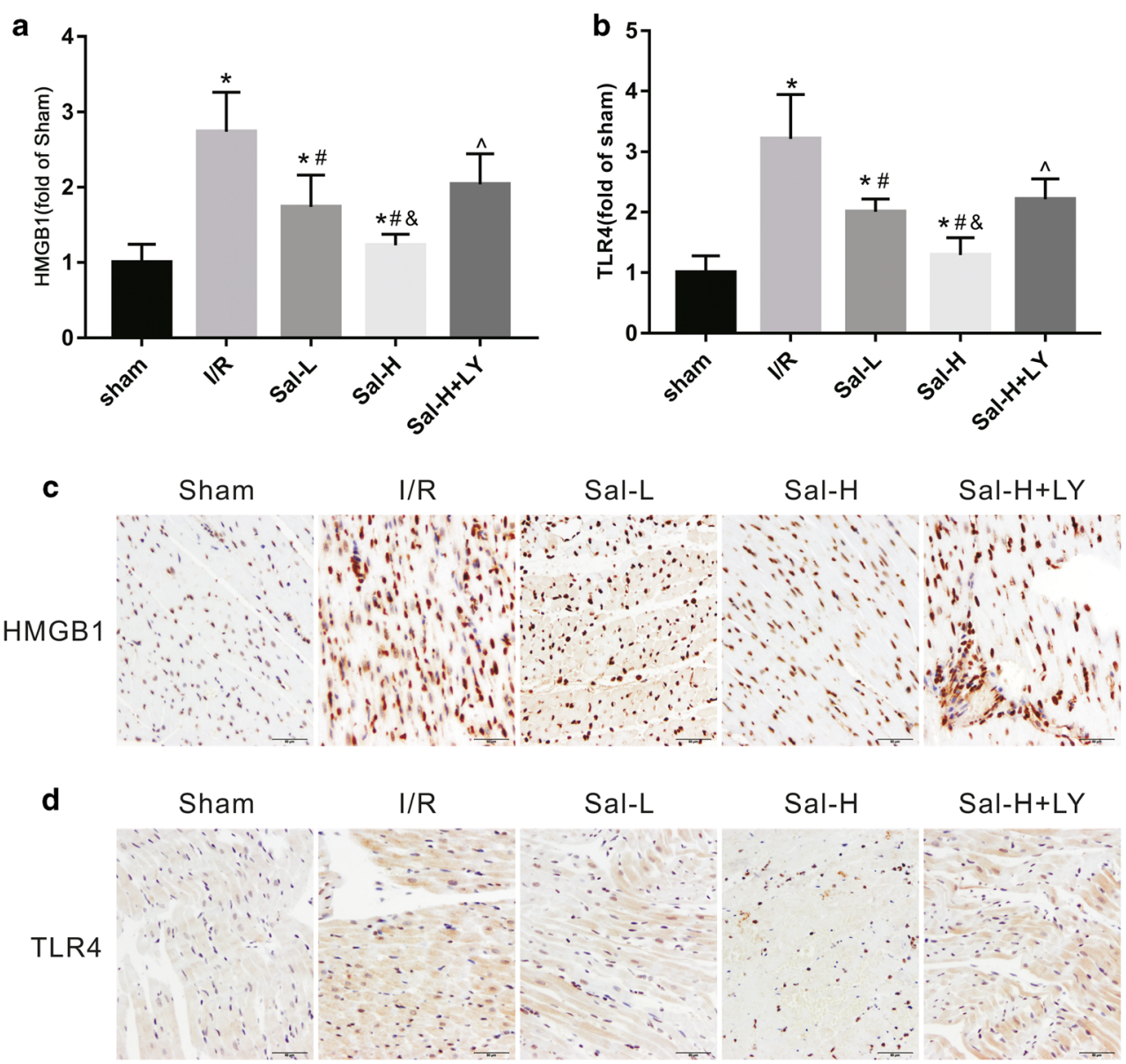

and increase the blood supply of the coronary artery. These results suggested that Sal B could improve cardiac function and reduce the infarction size in $\mathrm{I} / \mathrm{R}$ model rats. Therefore, further investigation will be necessary to dissect the mechanisms for the potential beneficial clinical applications of Sal B.

Myocardial I/R injury is a complex pathological process, involving various potential mechanisms. It has been proven that myocardial inflammation and apoptosis are the main factors in myocardial I/R injury (Qiao and Xu 2016; Zhang, Liu and Geng 2018). It was also reported that inflammatory cytokines can damage myocardium and may even lead to a systemic inflammatory response (Trachtenberg and Hare 2017).

During myocardial I/R injury, various pro-inflammatory factors are released, which leads to the release of inflammatory mediators such as interleukin (IL) and TNF- $\alpha$, which can also release more inflammatory cytokines, cause inflammatory response and aggravate myocardial injury (Kalogeris et al. 2016). Previous studies have shown that myocardial I/R injury can induce the release of inflammatory cytokines such as TNF- $\alpha$, IL-6 and HMGB1 (Wang et al. 2013; Hu et al. 2014) (Fig. 7a). We examined the expression of TNF- $\alpha$, IL18, IL-1 $\beta$ and HMGB1 (Fig. 7b) and found that Sal B could inhibit the level of these myocardial inflammatory cytokines. This result demonstrated that Sal B may protect against myocardial injury by reducing the elevation of these inflammatory cytokines.

In addition, cardiomyocyte apoptosis is considered to be a key factor leading to myocardial I/R injury. Some studies have shown that the role of the $\mathrm{Bcl}-2$ protein family is crucial in the process of cell apoptosis gene regulation (Xie et al. 2001) (Fig. 7a). We detected the expression of pro-apoptotic (Bax) and anti-apoptotic factors (Bcl-2) as well as the apoptosis rate in each experimental group. In our study, the expression of Bcl-2 significantly decreased, while the Bax expression remarkably increased in the I/R group (Fig. 7b). The results showed that Bcl-2 and Bax play different roles in myocardial I/R injury. The experimental results also showed that the expression of Bax decreased and the expression of Bcl-2 increased after treatment with Sal B, indicating that Sal B can mediate the expression of apoptosis-related genes and exert an antiapoptotic role in cardiomyocytes. However, the antiapoptotic effect of Sal B was partly reversed by LY294002 treatment, suggesting that the regulation of apoptotic expression was related to the PI3K/Akt signalling pathway (Fig. 7b).

HMGB1, as a nuclear protein released by necrotic or apoptotic cells, is a novel pro-inflammatory cytokine (Scaffidi, Misteli and Bianchi 2002; Bell et al. 2006). Under pathological conditions, HMGB1 can be actively or passively secreted 
Fig. 5 Myocardial apoptosis. Sal B suppressed myocardial apoptosis (a) in the I/R group. However, this anti-apoptotic effect was reversed by the administration of LY294002. The rate of apoptotic cells (b) was analysed with TUNEL kit and the expression levels of Bcl-2 protein (c and f) and Bax protein (d and f) and the ratio of $\mathrm{Bcl}-2 / \mathrm{Bax}(\mathbf{e})$ were measured with western blotting. All data are expressed as mean \pm $\mathrm{SD},{ }^{*} P<0.05$ versus Sham, ${ }^{\#} P<$ 0.05 versus $\mathrm{I} / \mathrm{R},{ }^{\&} P<0.05$ versus Sal-L, ${ }^{\wedge} P<0.05$ versus Sal-H

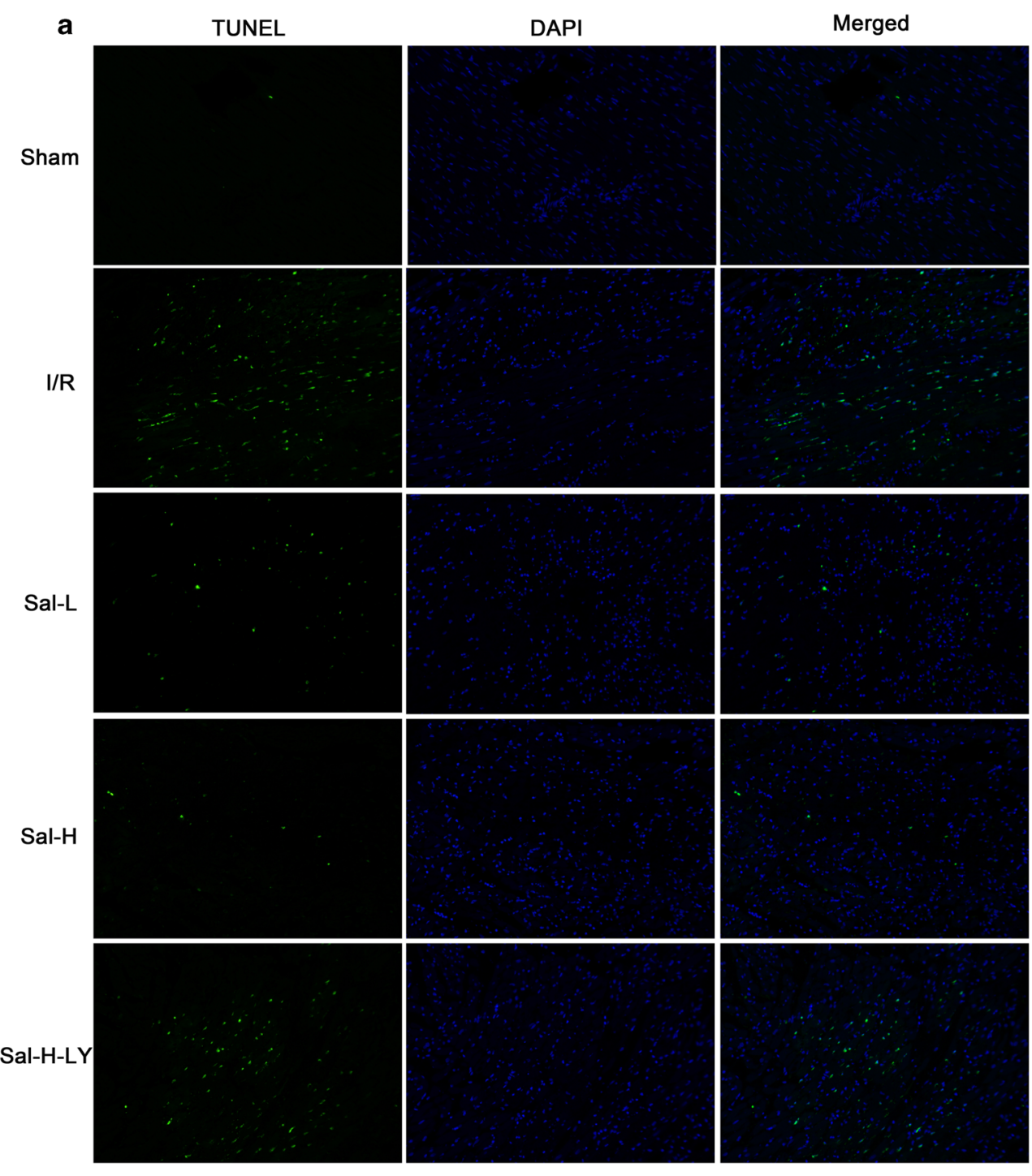

outside of the cell, promoting inflammatory response (Scaffidi et al. 2002; Yamada and Maruyama 2007). Some recent research has shown that HMGB1 plays an important role in myocardial I/R injury by regulating inflammatory factors (Andrassy et al. 2008; Hu et al. 2010; Wang et al. 2012). More recent studies have reported that the inhibition of HMGB1 expression by minocycline and dobutamine alleviates inflammatory responses and myocardium apoptosis in myocardial I/R injury (Wang et al. 2013; Cheng et al. 2018).

TLR4 (receptor of HMGB1), as an inflammatory factor, has been shown to play a detrimental role in myocardial $\mathrm{I} / \mathrm{R}$ injury (Wang et al. 2016; Yang et al. 2018). Studies have also shown that the activation of TLR4 can aggravate myocardial inflammation and the effective inhibition of TLR4 may be the key to preventing adverse myocardial remodelling (Vilahur and Badimon 2014; Lee, Hutchinson and Saint 2016). In our present study, we found that the expression of HMGB1 and TLR4 was significantly increased in the I/R group but sharply was decreased with Sal B treatment. Based on the above studies and our current findings, we speculated that HMGB1 and TLR4 may promote the inflammatory response in myocardial $\mathrm{I} / \mathrm{R}$ injury and that Sal B could inhibit myocardial $\mathrm{I} / \mathrm{R}$ injury through the inhibition of HMGB1 and TLR4 expression.

$\mathrm{PI} 3 \mathrm{~K} / \mathrm{Akt}$ is a well-known signalling pathway involved in myocardial protection (Hausenloy and Yellon 2004). Several studies have shown that the activation of the PI3K/Akt signalling pathway attenuated myocardial I/R injury by inhibiting the inflammatory response, endoplasmic reticulum stressregulated apoptosis and autophagy (Xu et al. 2018; Ye et al. 2018; Yu et al. 2019). However, it was unclear whether the $\mathrm{PI} 3 \mathrm{~K} / \mathrm{Akt}$ pathway mediates the cardioprotective effects of Sal B.

In the present study, we found that after adding LY294002 treatment to the group treated with a high dose of Sal B, the size of myocardial infarction, the levels of myocardial necrosis and myocardial inflammation markers and the expression 

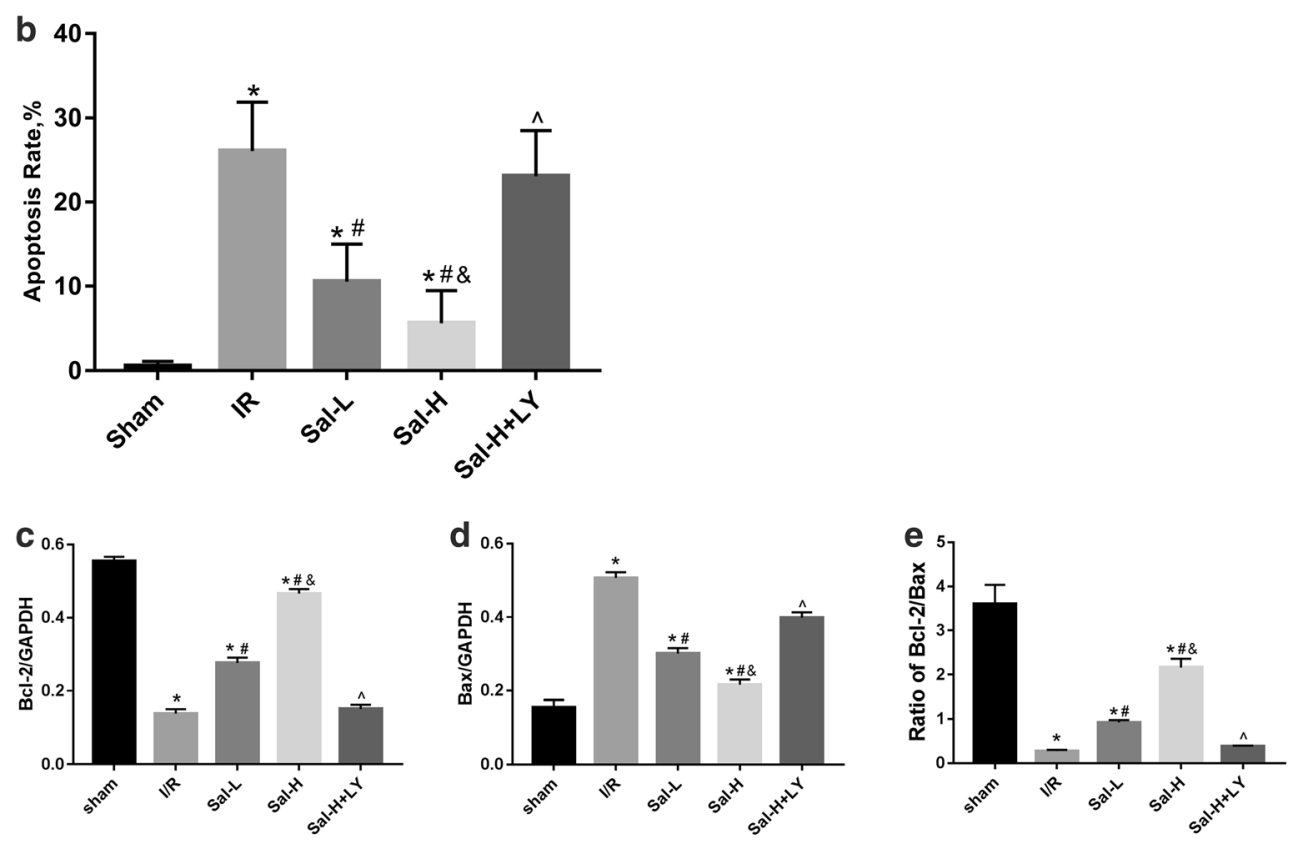

f

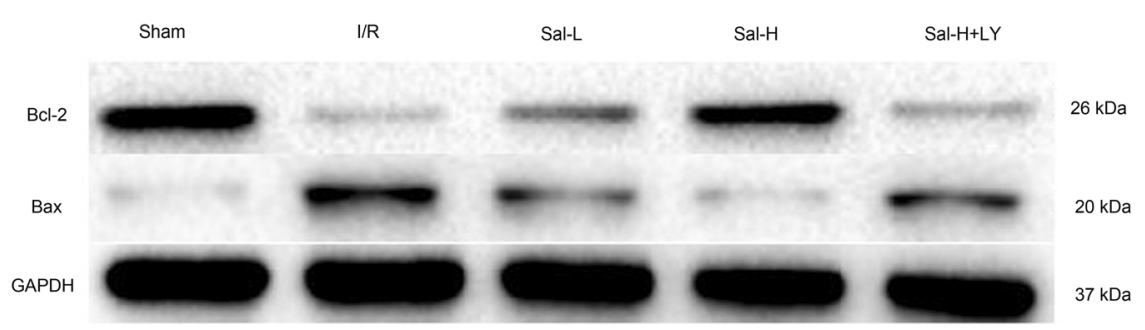

Fig. 5 (continued)

of HMGB1 were significantly increased. These results indicated that the cardioprotective effect of Sal B was reversed by LY294002 and that the cardioprotective mechanism of Sal B was involved in the activation of the PI3K/Akt signalling pathway and the inhibition of HMGB1 expression.

It is reported that rats and humans have highly similar genomes, with a similarity of $90 \%$. Its heart structure is also highly similar to that of humans (Gibbs et al. 2004). Previous reports have indicated that inflammation and apoptosis contribute to the initiation and development of I/R injury (Nakano et al. 2016, Schubert et al. 2016). It is confirmed that the most robust end point in experimental animal studies on cardioprotection is infarct size (Botker et al. 2018; Lindsey et al. 2018) and cardioprotective effects beyond the immediate reduction of infarct size are thus not considered, that is, on delayed cell death (by apoptosis or autophagy rather than necrosis), inflammation, repair, remodelling, all of which are, however, essential for clinical outcome studies (Jones et al. 2015). Our result proved Sal B reduced myocardial infarction size by means of reducing myocardial injury and inflammatory response and suppressing myocardial apoptosis.

To validate Sal B could improve cardiac function and reduce the infarction size in $\mathrm{I} / \mathrm{R}$ model rats, further studies focused on pathological mechanisms and therapeutic reactions are worthy of investigation. We must admit that in the field of cardioprotection, substantial gaps remain between experimental studies aiming at the identification of novel mechanisms and studies providing robust preclinical data which is worth to be tested in humans. Despite the advances represented here, we are clearly still at the beginning of the translation from numerous successful animal experiments to clinical practice on cardioprotection. Much of the additional data needed to complete this story will come from other species, distantly related to rat. Future trials must focus on phase II dose and time data, and recruit patients who have truly a chance to benefit from cardioprotection.

\section{Study limitations}

Our results suggested that Sal B can attenuate myocardial I/R injury by reducing myocardial inflammation and apoptosis. And the cardioprotective mechanism of Sal B on myocardial I/R injury was through reducing the release of HMGB1 through activating the PI3K/Akt signalling pathway. Inevitably, the present study has shortcomings. Firstly, in the 
Fig. 6 PI3K/Akt/HMGB1 signalling pathway. Sal B activated the PI3K/Akt pathway and suppressed HMGB1 expression. But the effects were blocked by treatment with LY294002. Expression of T-Akt(11E7) (a and b), P-Akt(Ser473) (a and b) and HMGB1 (c and d) protein was measured with western blotting. All data are expressed as mean \pm $\mathrm{SD}, * P<0.05$ versus Sham, ${ }^{*} P<$ 0.05 versus $\mathrm{I} / \mathrm{R},{ }^{\&} P<0.05$ versus Sal-L, ${ }^{\wedge} P<0.05$ versus Sal-H
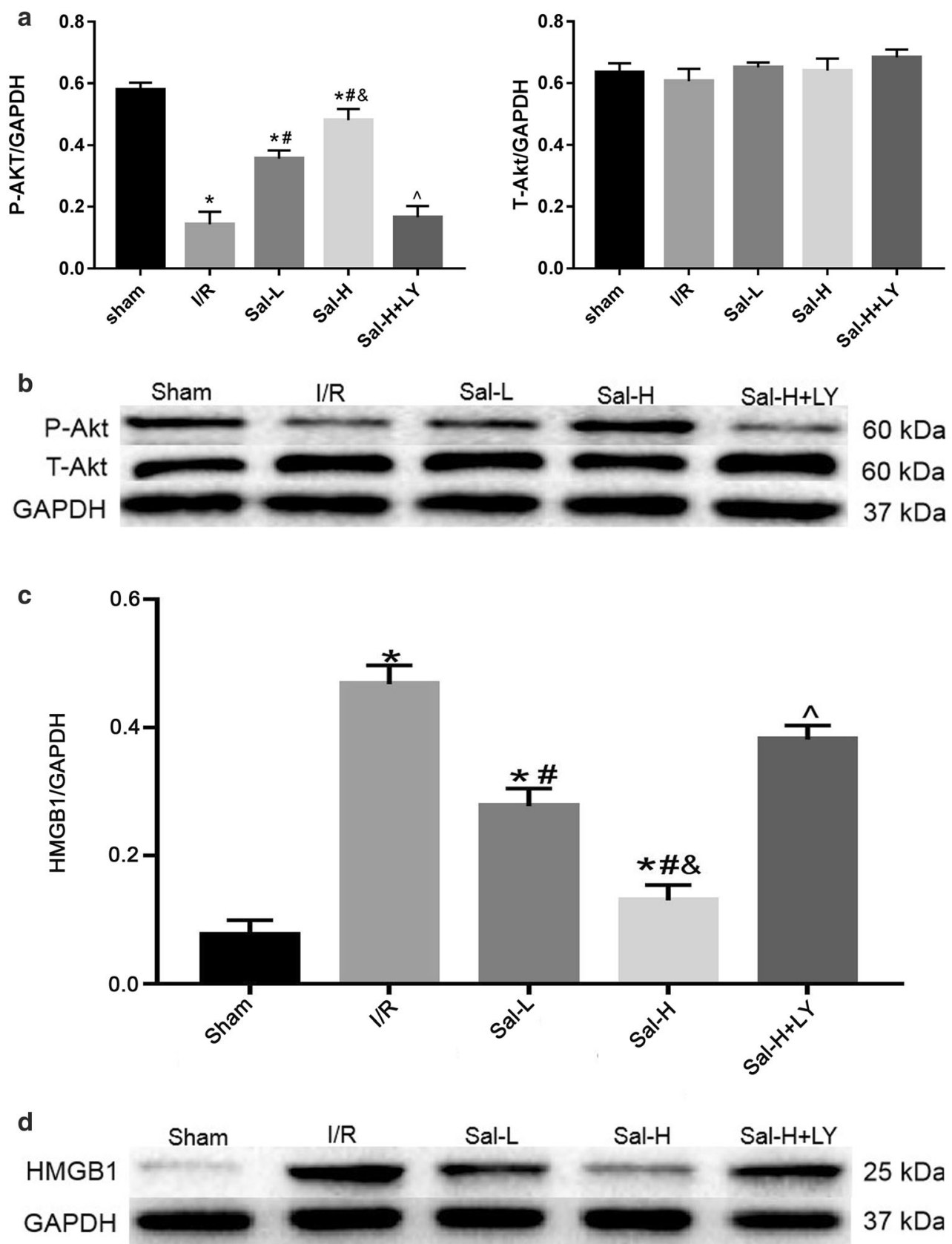

present study, quantification of inflammatory mediators was investigated at only one time point ( $24 \mathrm{~h}$ after reperfusion). Indeed, it is not clear whether myocardial I/R injury causes the response of myocardial inflammatory mediators or whether the release of inflammatory mediators leads to myocardial I/ $\mathrm{R}$ injury. Further research will be needed to determine the expression of these inflammatory factors in a timedependent manner. Secondly, when assessing myocardial infarct size, the experimental criterion method is the blue dye coloration for delineating infarct area at risk and the identification of viable myocardium by TTC. However, we did not determine the area at risk using blue staining in our experiment. Thirdly, our study provides a potential mechanism for myocardial protection in myocardial I/R injury. However, in the absence of any intervention, different animals, animals of different ages, diets, sources, different laboratory operations and the outcome will be affected. The infarct size of mice, rabbits and pigs can be significantly reduced after ischemic preconditioning, but the release time of troponin is different (Jones et al. 2015). There are significant differences among different species. It is not enough for us to study only one animal model of I/R. Fourthly, it is undeniable that experimental studies in rats can provide reliable preclinical data and deserve to be tested in humans. But this program is to consequence of the high cost of converting rat data to humans/ patient data, and the pressing need to further improved and 

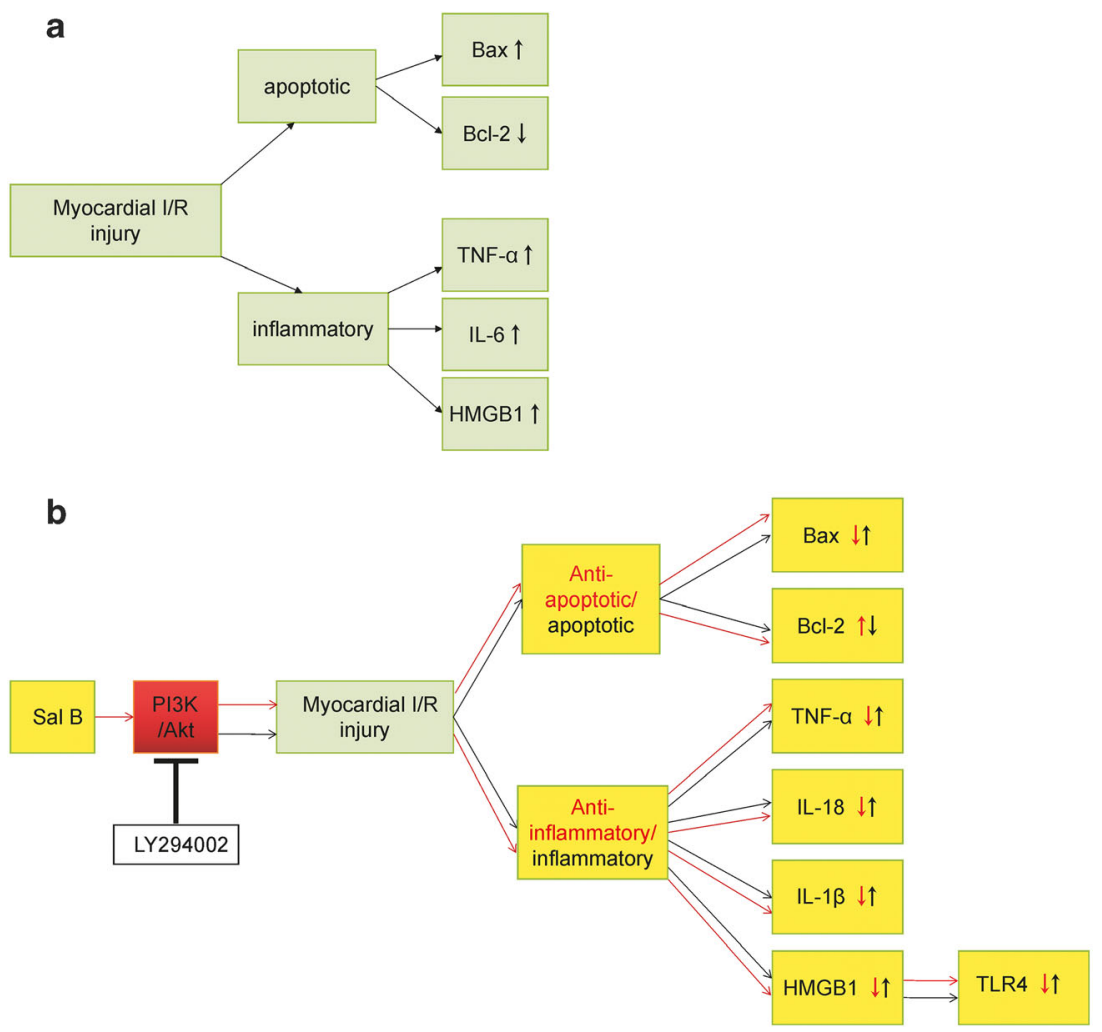

Fig. 7 The hypothesis that Sal B protects myocardium through inhibiting the expression of HMGB1 via activating PI3k/Akt signalling pathway in myocardial I/R injury. Previous studies reported that myocardial I/R injury can induce the release of inflammatory cytokines such as TNF- $\alpha$, IL6 and HMGB1, and induce cardiomyocyte apoptosis, resulting in the decrease of Bcl-2 and the increase of Bax (a). In our study, Sal B alleviates myocardial inflammation by reducing the elevation of these inflammatory cytokines (TNF- $\alpha$, IL-18, IL-1 $\beta$, HMGB1 and TLR4) during myocardial I/R injury. Additionally, Sal B can inhibit cardiomyocyte

enhanced, such as adding the risk factors, considering the lack of adequate phase II dose and time studies and expanding the number of experimental subjects and scope. However, with the advent of low-cost rat experiments, it is possible to apply for research funding to expand the experiment to bring it closer to current human data.

\section{Conclusions}

The present study indicated that Sal B preconditioning attenuated myocardial I/R injury by reducing myocardial inflammation and apoptosis. In addition, our findings suggest that Sal B attenuated myocardial I/R injury by activating PI3K/Akt signalling pathway and inhibiting the release of HMGB1 in rats.

Author contributions Hanqing Liu and Wei Liu conceived, designed and performed most of the experiments, and analysed the data. Huiliang Qiu performed some parts of the research. Hanqing apoptosis by inhibiting the expression of Bcl and Bax. All effects of Sal B are reversed by LY294002 (b). In panel b, the red arrow represents the anti-inflammatory and anti-apoptotic effects of Sal B through activating PI3K/Akt pathway on myocardial I/R injury. The black arrow represents that the effect of Sal B is reversed after LY294002. Sal B, Salvianolic acid B. PI3k/Akt, phosphoinositide 3-kinase/protein, kinase B. HMGB1, high mobility group protein 1 . TNF- $\alpha$, tumour necrosis factor- $\alpha$. IL-18, interleukin-18. IL-1 $\beta$, interleukin-1 $\beta$. TLR4, toll-like receptor 4. Bcl-2, beclin-2. Bax, bcl-2 associated $\times$ protein. LY294002, an inhibitor of PI3K

Liu and Wei Liu wrote, and Danping Xu, Chaoyang Zheng, Dezhi Zou, Huayang Cai and Qiuxiong Chen revised the manuscript. The final version has been approved and agreed for publication by all authors and by the Scientific Research Office of Guangdong Provincial Hospital of Chinese Medicine.

Funding information This study was supported by Guangzhou Science and Technology Plan project (No. 201710010107), Guangdong Provincial Science and Technology Plan (No. 2016A020226011) and Joint Innovation Specific Project in Key Areas from Guangdong Branch Institute of China Academy of Chinese Medical Sciences (No. ZZ0908065).

\section{Compliance with ethical standards}

All animals used in this study were treated in accordance with the Regulations of Experimental Animal Administrations published by the State Committee of Science and Technology of the People's Republic of China. The study protocol was approved by the Animal Care Committee of Guangdong Provincial Hospital of Chinese Medicine.

Conflict of interest The authors declare that they have no conflict of interest. 
Open Access This article is distributed under the terms of the Creative Commons Attribution 4.0 International License (http:// creativecommons.org/licenses/by/4.0/), which permits unrestricted use, distribution, and reproduction in any medium, provided you give appropriate credit to the original author(s) and the source, provide a link to the Creative Commons license, and indicate if changes were made.

\section{References}

Andrassy M, Volz HC, Igwe JC, Funke B, Eichberger SN, Kaya Z, Buss S, Autschbach F, Pleger ST, Lukic IK, Bea F, Hardt SE, Humpert PM, Bianchi ME, Mairbaurl H, Nawroth PP, Remppis A, Katus HA, Bierhaus A (2008) High-mobility group box-1 in ischemiareperfusion injury of the heart. Circulation 117:3216-3226

Bell CW, Jiang W, Reich CF 3rd, Pisetsky DS (2006) The extracellular release of HMGB1 during apoptotic cell death. Am J Physiol Cell Physiol 291:C1318-C1325

Binder A, Ali A, Chawla R, Aziz HA, Abbate A, Jovin IS (2015) Myocardial protection from ischemia-reperfusion injury post coronary revascularization. Expert Rev Cardiovasc Ther 13:1045-1057

Botker HE, Hausenloy D, Andreadou I, Antonucci S, Boengler K, Davidson SM, Deshwal S, Devaux Y, Di Lisa F, Di Sante M, Efentakis P, Femmino S, Garcia-Dorado D, Giricz Z, Ibanez B, Iliodromitis E, Kaludercic N, Kleinbongard P, Neuhauser M, Ovize M, Pagliaro P, Rahbek-Schmidt M, Ruiz-Meana M, Schluter KD, Schulz R, Skyschally A, Wilder C, Yellon DM, Ferdinandy P, Heusch G (2018) Practical guidelines for rigor and reproducibility in preclinical and clinical studies on cardioprotection. Basic Res Cardiol 113:39

Braunersreuther V, Montecucco F, Asrih M, Pelli G, Galan K, Frias M, Burger F, Quindere AL, Montessuit C, Krause KH, Mach F, Jaquet V (2013) Role of NADPH oxidase isoforms NOX1, NOX2 and NOX4 in myocardial ischemia/reperfusion injury. J Mol Cell Cardiol 64:99-107

Cao Z, Ren D, Ha T, Liu L, Wang X, Kalbfleisch J, Gao X, Kao R, Williams D, Li C (2013) CpG-ODN, the TLR9 agonist, attenuates myocardial ischemia/reperfusion injury: involving activation of PI3K/Akt signaling. Biochim Biophys Acta 1832:96-104

Chan K, Chui SH, Wong DY, Ha WY, Chan CL, Wong RN (2004) Protective effects of Danshensu from the aqueous extract of Salvia miltiorrhiza (Danshen) against homocysteine-induced endothelial dysfunction. Life Sci 75:3157-3171

Chen J, Jiang Z, Zhou X, Sun X, Cao J, Liu Y, Wang X (2019) Dexmedetomidine preconditioning protects cardiomyocytes against hypoxia/reoxygenation-induced necroptosis by inhibiting HMGB1mediated inflammation. Cardiovasc Drugs Ther 33:45-54

Cheng X, Hu J, Wang Y, Ye H, Li X, Gao Q, Li Z (2018) Effects of dexmedetomidine postconditioning on myocardial ischemia/ reperfusion injury in diabetic rats: role of the PI3K/Akt-dependent signaling pathway. J Diabetes Res 2018:3071959

Deng Y, Yang M, Xu F, Zhang Q, Zhao Q, Yu H, Li D, Zhang G, Lu A, Cho K, Teng F, Wu P, Wang L, Wu W, Liu X, Guo DA, Jiang B (2015) Combined salvianolic acid B and ginsenoside Rg1 exerts cardioprotection against ischemia/reperfusion injury in rats. PLoS One 10:e0135435

Fan Y, Luo Q, Wei J, Lin R, Lin L, Li Y, Chen Z, Lin W, Chen Q (2018) Mechanism of salvianolic acid B neuroprotection against ischemia/ reperfusion induced cerebral injury. Brain Res 1679:125-133

Frank A, Bonney M, Bonney S, Weitzel L, Koeppen M, Eckle T (2012) Myocardial ischemia reperfusion injury: from basic science to clinical bedside. Semin Cardiothorac Vasc Anesth 16:123-132
Gibbs RA, Weinstock GM, Metzker ML, Muzny DM, Sodergren EJ, Scherer S, Scott G, Steffen D, Worley KC, Burch PE, Okwuonu G, Hines S, Lewis L, DeRamo C, Delgado O, Dugan-Rocha S, Miner G, Morgan M, Hawes A, Gill R, Celera R, Holt A, Adams MD, Amanatides PG, Baden-Tillson H, Barnstead M, Chin S, Evans CA, Ferriera S, Fosler C, Glodek A, Gu Z, Jennings D, Kraft CL, Nguyen T, Pfannkoch CM, Sitter C, Sutton GG, Venter JC, Woodage T, Smith D, Lee HM, Gustafson E, Cahill P, Kana A, Doucette-Stamm L, Weinstock K, Fechtel K, Weiss RB, Dunn DM, Green ED, Blakesley RW, Bouffard GG, De Jong PJ, Osoegawa K, Zhu B, Marra M, Schein J, Bosdet I, Fjell C, Jones S, Krzywinski M, Mathewson C, Siddiqui A, Wye N, McPherson J, Zhao S, Fraser CM, Shetty J, Shatsman S, Geer K, Chen Y, Abramzon S, Nierman WC, Havlak PH, Chen R, Durbin KJ, Egan A, Ren Y, Song XZ, Li B, Liu Y, Qin X, Cawley S, Worley KC, Cooney AJ, D'Souza LM, Martin K, Wu JQ, Gonzalez-Garay ML, Jackson AR, Kalafus KJ, McLeod MP, Milosavljevic A, Virk D, Volkov A, Wheeler DA, Zhang Z, Bailey JA, Eichler EE et al (2004) Genome sequence of the Brown Norway rat yields insights into mammalian evolution. Nature 428:493-521

Han QF, Wu L, Zhou YH, Wang LH, Zhang DY, Liu T, Yao HC (2015) Simvastatin protects the heart against ischemia reperfusion injury via inhibiting HMGB1 expression through PI3K/Akt signal pathways. Int J Cardiol 201:568-569

Hausenloy DJ, Yellon DM (2004) New directions for protecting the heart against ischaemia-reperfusion injury: targeting the reperfusion injury salvage kinase (RISK)-pathway. Cardiovasc Res 61:448-460

Ho JH, Hong CY (2011) Salvianolic acids: small compounds with multiple mechanisms for cardiovascular protection. J Biomed Sci 18:30

Hu P, Luo GA, Zhao Z, Jiang ZH (2005) Quality assessment of radix salviae miltiorrhizae. Chem Pharm Bull (Tokyo) 53:481-486

Hu X, Zhou X, He B, Xu C, Wu L, Cui B, Wen H, Lu Z, Jiang H (2010) Minocycline protects against myocardial ischemia and reperfusion injury by inhibiting high mobility group box 1 protein in rats. Eur J Pharmacol 638:84-89

Hu G, Huang X, Zhang K, Jiang H, Hu X (2014) Anti-inflammatory effect of B-type natriuretic peptide postconditioning during myocardial ischemia-reperfusion: involvement of PI3K/Akt signaling pathway. Inflammation 37:1669-1674

Jennings RB (2013) Historical perspective on the pathology of myocardial ischemia/reperfusion injury. Circ Res 113:428-438

Ji XY, Tan BK, Zhu YZ (2000) Salvia miltiorrhiza and ischemic diseases. Acta Pharmacol Sin 21:1089-1094

Jones SP, Tang XL, Guo Y, Steenbergen C, Lefer DJ, Kukreja RC, Kong M, Li Q, Bhushan S, Zhu X, Du J, Nong Y, Stowers HL, Kondo K, Hunt GN, Goodchild TT, Orr A, Chang CC, Ockaili R, Salloum FN, Bolli R (2015) The NHLBI-sponsored consortium for preclinicAl assESsment of cARdioprotective therapies (CAESAR): a new paradigm for rigorous, accurate, and reproducible evaluation of putative infarct-sparing interventions in mice, rabbits, and pigs. Circ Res 116: $572-586$

Kalogeris T, Baines CP, Krenz M, Korthuis RJ (2016) Ischemia/reperfusion. Compr Physiol 7:113-170

Lam FF, Yeung JH, Chan KM, Or PM (2007) Relaxant effects of danshen aqueous extract and its constituent danshensu on rat coronary artery are mediated by inhibition of calcium channels. Vasc Pharmacol 46: 271-277

Lee SM, Hutchinson M, Saint DA (2016) The role of Toll-like receptor 4 (TLR4) in cardiac ischaemic-reperfusion injury, cardioprotection and preconditioning. Clin Exp Pharmacol Physiol 43:864-871

Li Y, Li J, Hou Z, Yu Y, Yu B (2016a) KLF5 overexpression attenuates cardiomyocyte inflammation induced by oxygen-glucose deprivation/reperfusion through the PPARgamma/PGC-1alpha/ TNF-alpha signaling pathway. Biomed Pharmacother 84:940-946 
Li D, Wang J, Hou J, Fu J, Liu J, Lin R (2016b) Salvianolic acid B induced upregulation of miR-30a protects cardiac myocytes from ischemia/reperfusion injury. BMC Complement Altern Med 16:336

Li X, Hu X, Wang J, Xu W, Yi C, Ma R, Jiang H (2018) Inhibition of autophagy via activation of PI3K/Akt/mTOR pathway contributes to the protection of hesperidin against myocardial ischemia/ reperfusion injury. Int J Mol Med 42:1917-1924

Lindsey ML, Bolli R, Canty JM Jr, Du XJ, Frangogiannis NG, Frantz S, Gourdie RG, Holmes JW, Jones SP, Kloner RA, Lefer DJ, Liao R, Murphy E, Ping P, Przyklenk K, Recchia FA, Schwartz Longacre L, Ripplinger CM, Van Eyk JE, Heusch G (2018) Guidelines for experimental models of myocardial ischemia and infarction. Am J Physiol Heart Circ Physiol 314:H812-H838

Lv H, Wang L, Shen J, Hao S, Ming A, Wang X, Su F, Zhang Z (2015) Salvianolic acid B attenuates apoptosis and inflammation via SIRT1 activation in experimental stroke rats. Brain Res Bull 115:30-36

Nakano Y, Matoba T, Tokutome M, Funamoto D, Katsuki S, Ikeda G, Nagaoka K, Ishikita A, Nakano K, Koga J, Sunagawa K, Egashira K (2016) Nanoparticle-mediated delivery of irbesartan induces cardioprotection from myocardial ischemia-reperfusion injury by antagonizing monocyte-mediated inflammation. Sci Rep 6:29601

Ouyang F, Huang H, Zhang M, Chen M, Huang H, Huang F, Zhou S (2016) HMGB1 induces apoptosis and EMT in association with increased autophagy following $\mathrm{H} / \mathrm{R}$ injury in cardiomyocytes. Int J Mol Med 37:679-689

Qiao Z, Xu Y (2016) Salvianolic acid B alleviating myocardium injury in ischemia reperfusion rats. Afr J Tradit Complement Altern Med 13: 157-161

Samsamshariat SA, Samsamshariat ZA, Movahed MR (2005) A novel method for safe and accurate left anterior descending coronary artery ligation for research in rats. Cardiovasc Revasc Med 6:121-123

Scaffidi P, Misteli T, Bianchi ME (2002) Release of chromatin protein HMGB1 by necrotic cells triggers inflammation. Nature 418:191195

Schubert C, Raparelli V, Westphal C, Dworatzek E, Petrov G, Kararigas G, Regitz-Zagrosek V (2016) Reduction of apoptosis and preservation of mitochondrial integrity under ischemia/reperfusion injury is mediated by estrogen receptor beta. Biol Sex Differ 7:53

Tang L, Mo Y, Li Y, Zhong Y, He S, Zhang Y, Tang Y, Fu S, Wang X, Chen A (2017) Urolithin A alleviates myocardial ischemia/ reperfusion injury via PI3K/Akt pathway. Biochem Biophys Res Commun 486:774-780

Tong S, Zhang L, Joseph J, Jiang X (2018) Celastrol pretreatment attenuates rat myocardial ischemia/ reperfusion injury by inhibiting high mobility group box 1 protein expression via the PI3K/Akt pathway. Biochem Biophys Res Commun 497:843-849

Trachtenberg BH, Hare JM (2017) Inflammatory cardiomyopathic syndromes. Circ Res 121:803-818

Vilahur G, Badimon L (2014) Ischemia/reperfusion activates myocardial innate immune response: the key role of the toll-like receptor. Front Physiol 5:496

Wang N, Min X, Li D, He P, Zhao L (2012) Geranylgeranylacetone protects against myocardial ischemia and reperfusion injury by inhibiting high-mobility group box 1 protein in rats. Mol Med Rep 5:521-524

Wang J, Yang H, Hu X, Fu W, Xie J, Zhou X, Xu W, Jiang H (2013) Dobutamine-mediated heme oxygenase-1 induction via PI3K and p38 MAPK inhibits high mobility group box 1 protein release and attenuates rat myocardial ischemia/reperfusion injury in vivo. J Surg Res 183:509-516
Wang YH, Chen KM, Chiu PS, Lai SC, Su HH, Jan MS, Lin CW, Lu DY, Fu YT, Liao JM, Chang JT, Huang SS (2016) Lumbrokinase attenuates myocardial ischemia-reperfusion injury by inhibiting TLR4 signaling. J Mol Cell Cardiol 99:113-122

Wang EW, Han YY, Jia XS (2018) PAFR-deficiency alleviates myocardial ischemia/reperfusion injury in mice via suppressing inflammation, oxidative stress and apoptosis. Biochem Biophys Res Commun 495:2475-2481

Wu N, Li W, Shu W, Jia D (2014) Protective effect of picroside II on myocardial ischemia reperfusion injury in rats. Drug Des Devel Ther 8:545-554

Xie Z, Koyama T, Suzuki J, Fujii Y, Togashi H, Sawa H, Nagashima K (2001) Coronary reperfusion following ischemia: different expression of bcl-2 and bax proteins, and cardiomyocyte apoptosis. Jpn Heart J 42:759-770

Xu T, Qin G, Jiang W, Zhao Y, Xu Y, Lv X (2018) 6-Gingerol protects heart by suppressing myocardial ischemia/reperfusion induced inflammation via the PI3K/Akt-dependent mechanism in rats. Evid Based Complement Alternat Med 2018:6209679

Xue L, Wu Z, Ji XP, Gao XQ, Guo YH (2014) Effect and mechanism of salvianolic acid $\mathrm{B}$ on the myocardial ischemia-reperfusion injury in rats. Asian Pac J Trop Med 7:280-284

Yamada S, Maruyama I (2007) HMGB1, a novel inflammatory cytokine. Clin Chim Acta 375:36-42

Yang J, Yang C, Yang J, Ding J, Li X, Yu Q, Guo X, Fan Z, Wang H (2018) RP105 alleviates myocardial ischemia reperfusion injury via inhibiting TLR4/TRIF signaling pathways. Int J Mol Med 41:32873295

Yao H, Han X, Han X (2014) The cardioprotection of the insulinmediated PI3K/Akt/mTOR signaling pathway. Am J Cardiovasc Drugs 14:433-442

Ye G, Fu Q, Jiang L, Li Z (2018) Vascular smooth muscle cells activate $\mathrm{PI} 3 \mathrm{~K} / \mathrm{Akt}$ pathway to attenuate myocardial ischemia/reperfusioninduced apoptosis and autophagy by secreting bFGF. Biomed Pharmacother 107:1779-1785

Yu Y, Xing N, Xu X, Zhu Y, Wang S, Sun G, Sun X (2019) Tournefolic acid B, derived from Clinopodium chinense (Benth.) Kuntze, protects against myocardial ischemia/reperfusion injury by inhibiting endoplasmic reticulum stress-regulated apoptosis via PI3K/AKT pathways. Phytomedicine 52:178-186

Zhang, L., J. Liu, T. Geng (2018) Ginkgetin aglycone attenuates the apoptosis and inflammation response through nuclear factor-kB signaling pathway in ischemic-reperfusion injury. $\mathrm{J}$ Cell Biochem

Zhao DH, Wu YJ, Liu ST, Liu RY (2017) Salvianolic acid B attenuates lipopolysaccharide-induced acute lung injury in rats through inhibition of apoptosis, oxidative stress and inflammation. Exp Ther Med 14:759-764

Zhou Y, Li Y, Mu T (2015) HMGB1 neutralizing antibody attenuates cardiac injury and apoptosis induced by hemorrhagic shock/ resuscitation in rats. Biol Pharm Bull 38:1150-1160

Zhu L, Wei T, Gao J, Chang X, He H, Luo F, Zhou R, Ma C, Liu Y, Yan T (2015) The cardioprotective effect of salidroside against myocardial ischemia reperfusion injury in rats by inhibiting apoptosis and inflammation. Apoptosis 20:1433-1443

Publisher's note Springer Nature remains neutral with regard to jurisdictional claims in published maps and institutional affiliations. 